\title{
REAL-TIME HERMITICITY DETECTION USING TITANIUM DIOXIDE THIN FILM RESISTOR AS BOTH OXYGEN AND PRESSURE SENSORS
}

\author{
Firas Sammoura* and Ken Yang
}

The Micromachined Product Division, Analog Devices, Wilmington, Massachusetts, USA

\begin{abstract}
In-situ hermiticity detection using Titanium dioxide $\left(\mathrm{TiO}_{2}\right)$ thin film resistors has been investigated for the first time. Titanium dioxide is widely used as an oxygen sensor, with accurate partial pressure reading at high temperatures. In addition to being oxygen sensitive at room temperature, $\mathrm{TiO}_{2}$ thin film resistors were found to be excellent pressure gauges at typical bonding pressures in the range of 1 Torr to 760 Torr. Combining the pressure sensitivity with the oxygen selectivity, Titanium dioxide thin film resistors constitute perfect hermiticity solutions for MEMS devices capped in an oxygen deficient environment at sub-atmospheric pressures. This unprecedented demonstration opens up new opportunities for monitoring real-time hermiticity in packaged MEMS devices, understanding the various failure modes during operation, and implementing the necessary diagnostic solutions.
\end{abstract}

\section{INTRODUCTION}

Hermiticity constitutes a critical precursor for the reliability of MEMS sensors. Ensuring hermiticity is a mandatory requirement for most encapsulated MEMS devices including automotive-grade sensors. Hermiticity failures emanates from two major sources: (1) Defects in the bonding material and (2) Cracks due to stress. A breach in hermiticity can change the gas content and pressure surrounding the MEMS device which in turns leads to performance shifts. In our previous work [1], we have found that any slight modification in the water content of the capped MEMS devices can lead to failure due to stiction. In addition, it is reported that the performance of RF MEMS switches is deleteriously affected when the devices are non-hermitically sealed due to the effect of humidity and small particles [2].

The state of the art hermiticity detection technique is Helium presence determination using mass spectroscopy [3]. However, this technique is not suitable for micro-cavities due to fast Helium diffusion rate. Several hermiticity detection techniques have been investigated. Elger et al. have investigated hermiticity existence optically using a non-contact profilometer with a chromatic confocal sensor. The pressure change inside the sealed cavity due to gas leakage causes a built-in membrane to deflect [4]. Pinter et. al have developed the technique of measuring the gas viscosity inside the cavity by the Quality factor (Q) of a vibratory device built inside the sealed MEMS devices [5]. Any breach in hermiticity will cause viscosity to change which is consequently detected by Quality factor shift. In addition, Lellouchi et al. adopted a Fourier transform infrared spectroscopy (FTIR) to quantify the concentration and composition of the gases in the sealed cavity [6]. These techniques do not allow for monitoring hermiticity in real-time during use and require expensive analysis equipment.

Titanium dioxide is an intrinsic semiconductor whose conductivity varies with the change in the concentration of the oxygen gas in the surrounding environment when deposited in the Anastase phase [7]. In this work, Titanium dioxide thin films are used to quantify the pressure and oxygen content of the sealant gas by simple resistance measurement enabling real-time hermiticity checking both during manufacturing and in-operation. In contrast to the previous publications, this is the first paper that investigates in-situ hermiticity detection of sealed MEMS devices using $\mathrm{TiO}_{2}$ thin film resistors integrated inside the MEMS cavity for devices capped in a sub- atmospheric pressure and in an oxygen deficient environment.

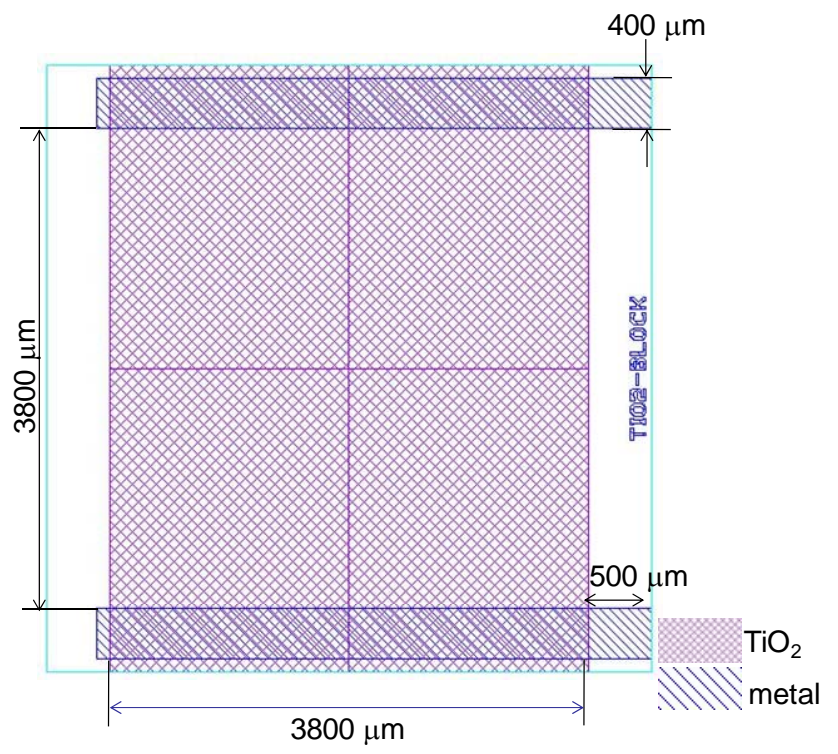

Figure 1: Layout schematic of a $\mathrm{TiO}_{2}$ test structure.

\section{FABRICATION PROCESS}

A Titanium dioxide thin film with metal electrodes was built on insulated substrate. The test structure was measured in a sealed pressure chamber that mimics the environment within a sealed MEMS device in order to validate the proposed hermiticity detection concept. Figure 1 shows the layout schematic of a $3800 \mu \mathrm{m} \times 3800 \mu \mathrm{m} \mathrm{TiO}_{2}$ block test structure. The fabrication process starts by thermally growing $1.47 \mu \mathrm{m}$ of oxide followed by the deposition of $1200 \AA$ of stoichiometric nitride to form the insulation layers. A $2200 \AA$ thick Titanium dioxide film was then deposited on the wafer using Plasma Enhanced Chemical Vapor Deposition (PECVD), and consequently annealed at $500^{\circ} \mathrm{C}$ for $30 \mathrm{~min}$ in a vacuum environment in order to form the Anastase phase [8]. The $\mathrm{TiO}_{2}$ film was then patterned with a wet etchant composed of $5: 1: 4 \mathrm{HNO}_{3}$ : $\mathrm{HF}$ : $\mathrm{H} 2 \mathrm{O}$ at $70^{\circ} \mathrm{C}$. A $200 \AA / 2000 \AA$ of $\mathrm{Ti} / \mathrm{Au}$ was then deposited and patterned by lift-off to form metal contacts to the $\mathrm{TiO}_{2}$ film resistor.

The integration of the $\mathrm{TiO}_{2}$ thin film resistors inside a capped MEMS sensor is proposed to be achieved using Through Silicon Via (TSV) technology as shown in the process flow outlined in Figure 2. The $\mathrm{TiO}_{2}$ thin film is deposited on the cap side of the sealed MEMS devices. The processing of the cap wafer starts by forming front side alignment marks on the front side of the wafer. Vias are then etched on the back side of the wafer up to a depth of two-thirds the total wafer thickness using Deep Reactive Ion Etching (DRIE). Via isolation is achieved by a thin thermally grown oxide of $250 \mathrm{~nm}$ in thickness. The cavity is later filled with a thin conductor seed layer followed by copper electroplating. The deposited copper is grinded down to the underlying oxide liner 
using Chemical Mechanical Polishing (CMP). Titanium dioxide is deposited on the back side of the wafer and the front side is grinded down using CMP to get access to the copper bond pads. The cap wafer is finally bonded to the device wafer.

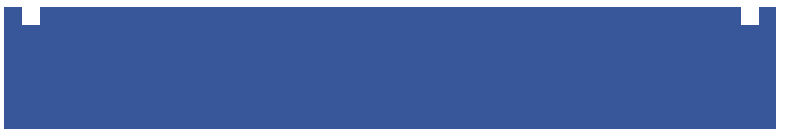

(a) front side alignment mark

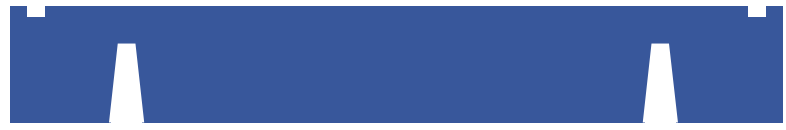

(b) via etch (DRIE)

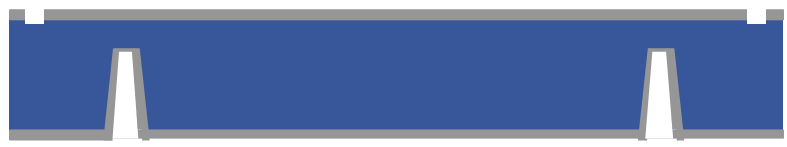

(c) via isolation (oxidation)

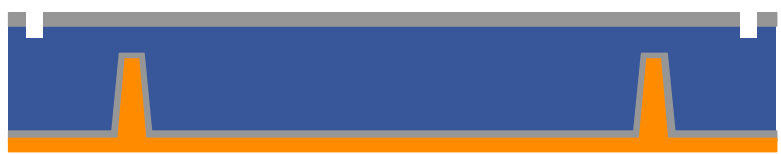

\section{(d) via fill with $\mathrm{Cu}$}

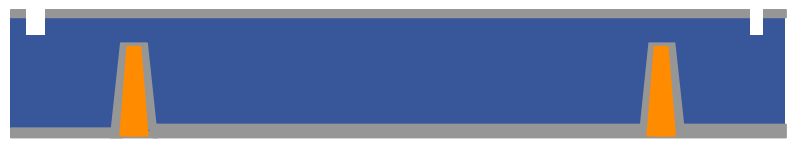

\section{(e) CMP Cu}

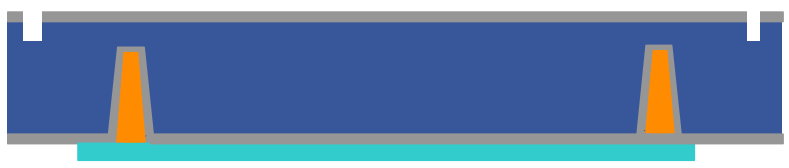

\section{(f) TiO2 deposition and etch}

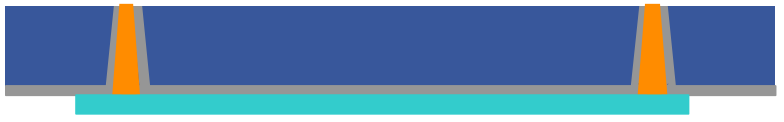

(g) CMP to expose Cu contacts

Figure 2: Fabrication Process of $\mathrm{TiO}_{2}$ thin film resistors on cap side using Through Silicon Via (TSV) technology.

\section{RESULTS AND DISCUSSION}

The thin film resistor were tested in a Lake Shore low temperature probe chamber Model CPX-HF equipped with a temperature controller Model 332 with built-in probes and gas inlets, where both $\mathrm{O}_{2}$ and $\mathrm{N}_{2}$ gases can be flown and chamber pressure was monitored as shown in Figure 3. An Agilent B1500A parametric semiconductor analyzer was used to measure the I-V curves necessary to extract the thin film resistivity where the voltage was swept between 0 and $1.5 \mathrm{~V}$ at an increment of $50 \mathrm{mV}$.

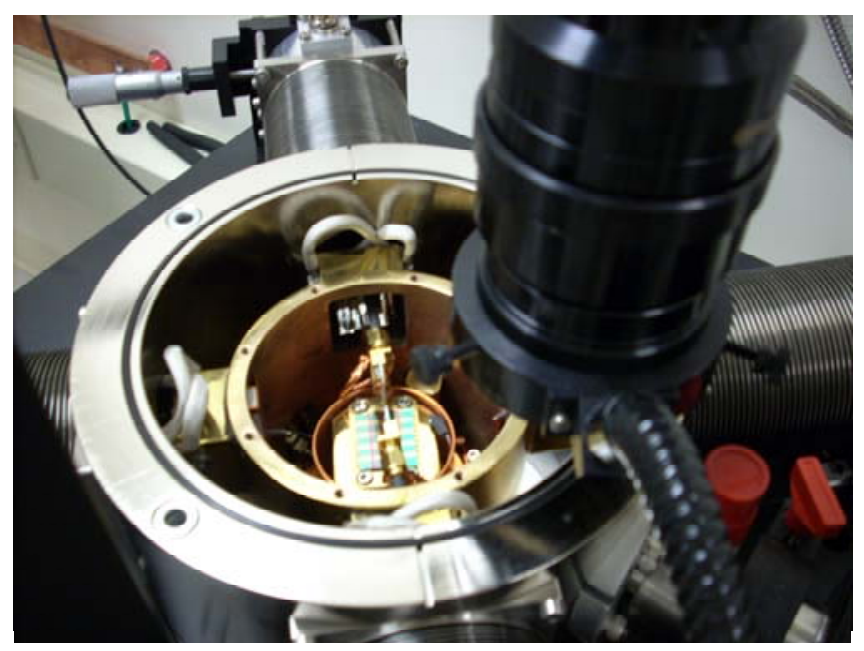

Figure 3: Digital picture showing testing setup of $\mathrm{TiO}_{2}$ thin film resistors inside an enclosed chamber.

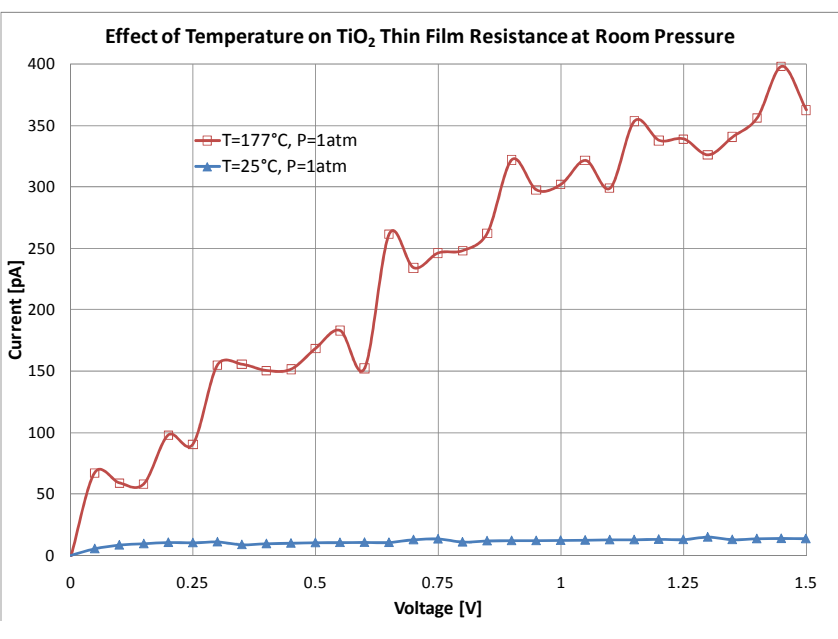

Figure 4: The $\mathrm{TiO}_{2}$ thin film resistance vs. temperature at atmospheric pressure.

The resistance of the Titanium dioxide thin film resistor was measured at both room temperature $\left(25^{\circ} \mathrm{C}\right)$ and $177^{\circ} \mathrm{C}$ as shown in Figure 4. Both tests were done are normal pressure of $1 \mathrm{~atm}$ with the chamber lid open. At room temperature the resistance of the thin film resistor as outlined in the schematic shown in Figure 1 was measured as $200 \mathrm{G} \Omega$. The resistivity can be then deduced as follows:

$$
R=\frac{\rho W t}{L}
$$

where $\mathrm{R}$ is the resistance of the thin film resistor, $\rho$ is the film resistivity, $\mathrm{W}$ is the width of the resistor with a value $3800 \mu \mathrm{m}$ for this resistor structure, $t$ is the thickness of the film with a value of $220 \mathrm{~nm}$, and $\mathrm{L}$ is the resistor length with a value of $3800 \mu \mathrm{m}$. As such the resistivity of the Titanium dioxide film at room temperature is calculated as $4.4 \times 10^{6} \Omega-\mathrm{cm}$. At $177^{\circ} \mathrm{C}$, the resistance is measured $4 \mathrm{G} \Omega$, and as such the resistivity is calculated as $88 \times 10^{3} \Omega-\mathrm{cm}$. The resistivity decreased 50 times as the chuck temperature increased from room temperature to $177^{\circ} \mathrm{C}$ 
at atmospheric pressure as can be inferred from the I-V curves shown in Figure 4 . The resistivity of sputtered thin film of Titanium dioxide has been reported in the literature with values of $4.95 \times 10^{6} \Omega$-cm and $20 \times 10^{3} \Omega-\mathrm{cm}$ at $25^{\circ} \mathrm{C}$ and $177^{\circ} \mathrm{C}$, respectively [9]. The value measured at room temperature is very close to the value reported in the literature. The discrepancy between the measured value and the value reported in the literature at $177^{\circ} \mathrm{C}$ is attributed to the lower equilibrium temperature of the Titanium dioxide thin film due to the silicon substrate and epoxy glue separation between the heated chuck and the tested sample.

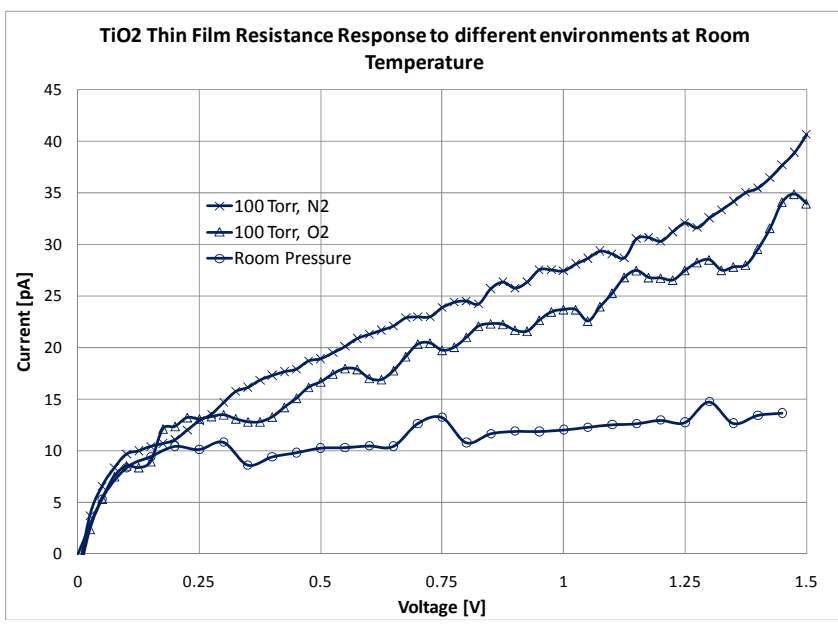

Figure 5: Response of $\mathrm{TiO}_{2}$ thin film resistors capped at 100 Torr of pure $\mathrm{N}_{2}, 100$ Torr of pure $\mathrm{O}_{2}$, and normal room pressure at room temperature of $25^{\circ} \mathrm{C}$.

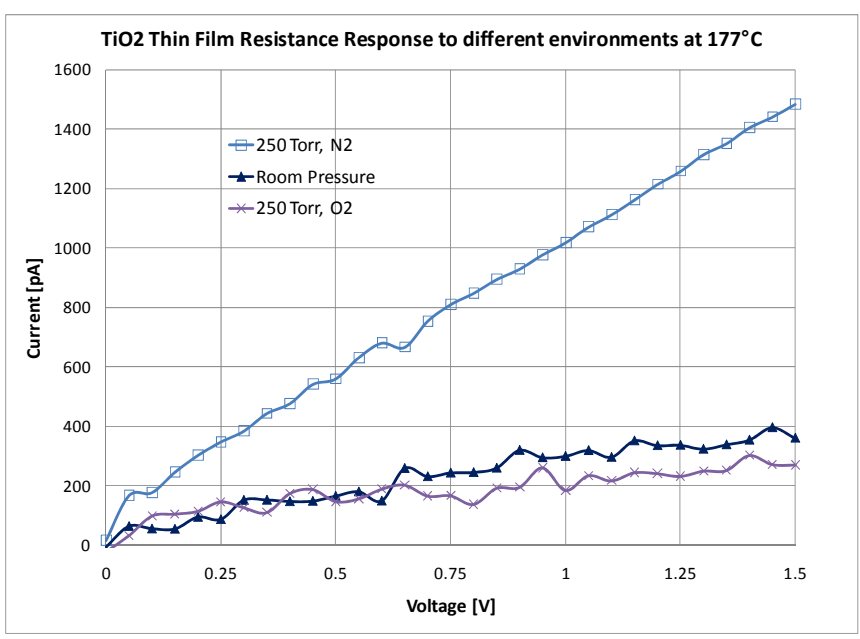

Figure 6: Response of $\mathrm{TiO}_{2}$ thin film resistors capped at 250 Torr of pure $\mathrm{N}_{2}, 250$ Torr of pure O2, and room pressure at a temperature of $177^{\circ} \mathrm{C}$.

In order to reproduce micro-cavity gas environment conditions, the chamber was evacuated and then filled with pure nitrogen gas $\left(\mathrm{N}_{2}\right)$ up to 100 Torr pressure, a typical gas content and pressure level of many capped MEMS devices. As shown in Figure 5, current flowing through the $\mathrm{TiO}_{2}$ thin film at $1.5 \mathrm{~V}$ DC Bias and room temperature dropped from 40pA at bonding environment to roughly 10pA when the control chamber was opened and exposed to atmospheric pressure. As such the resistance dropped 4 times as hermiticity was breached and sensor environment changed from pure Nitrogen at 100 Torr to a room temperature and pressure environment. The resistivity of the Titanium dioxide thin film was also measured at 100 Torr of pure Oxygen gas. There is a distinct shift in thin film resistivity as shown in Figure 5 with the Titanium dioxide resistance increasing from $48 \mathrm{G} \Omega$ in a Nitrogen environment to $58 \mathrm{G} \Omega$ in an Oxygen environment. This shows the selectivity of the thin film sensor to oxygen at room temperature.

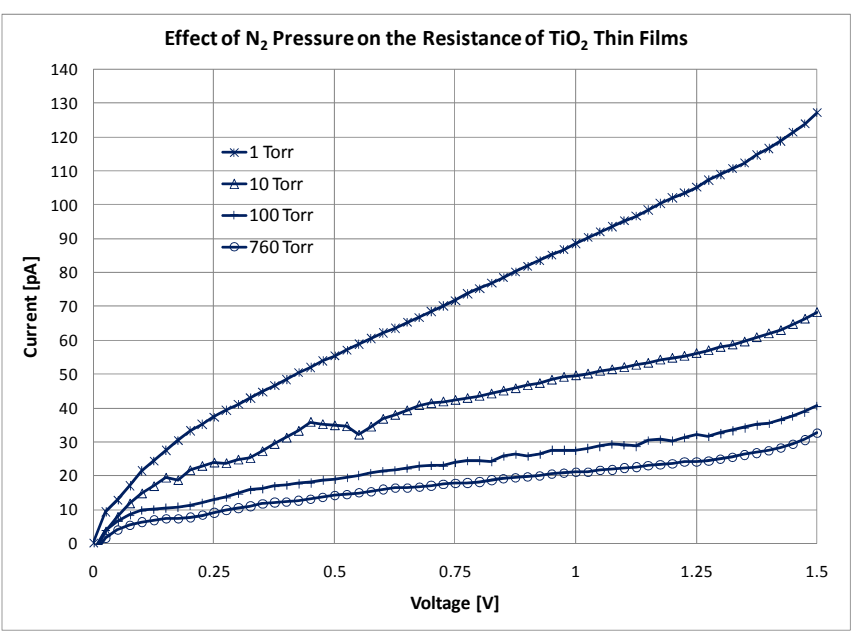

Figure 7: $\mathrm{TiO}_{2}$ thin film resistors $\mathrm{I}-\mathrm{V}$ curves for varying $\mathrm{N}_{2}$ pressures showing increase in resistance as $N_{2}$ pressure inside the control chamber increases.

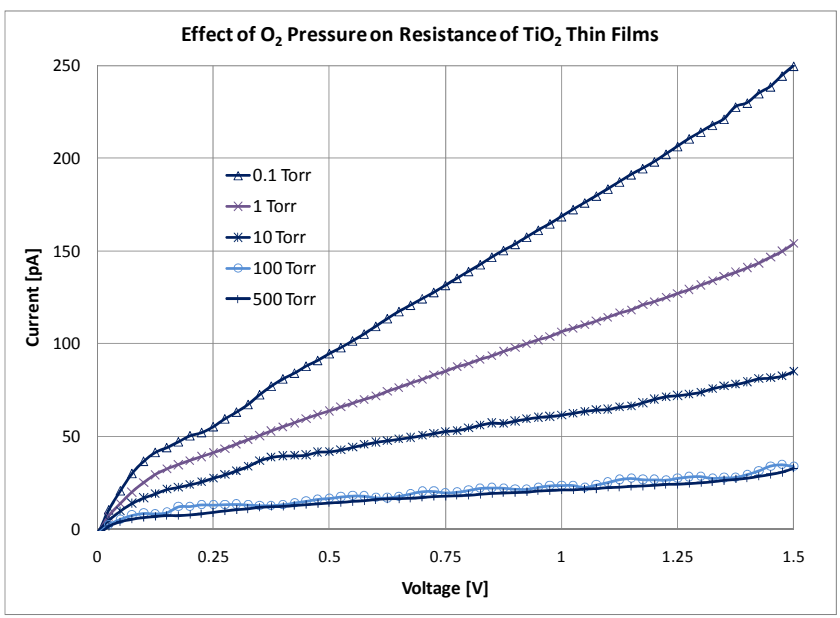

Figure 8: $\mathrm{TiO}_{2}$ thin film resistors $\mathrm{I}-\mathrm{V}$ curves for varying $\mathrm{O}_{2}$ pressures showing increase in resistance as $\mathrm{O}_{2}$ pressure inside the control chamber increases.

As the temperature increased to $177^{\circ} \mathrm{C}$, the current flowing through the $\mathrm{TiO}_{2}$ thin film dropped from 1480pA for a hermitically sealed die capped at 250 Torr of pure Nitrogen to 360pA for a leaky die exposed to atmospheric pressure as shown in Figure 6. This corresponds to a resistance drop from roughly $4 \mathrm{G} \Omega$ to $1 \mathrm{G} \Omega$ as hermiticity is breached. Response of $\mathrm{TiO}_{2}$ thin film resistors to pure $\mathrm{O}_{2}$ environment with same pressure as pure $\mathrm{N}_{2}$ capped devices is also measured. The current flowing through the $\mathrm{TiO}_{2}$ thin film resistor was $270 \mathrm{pA}$ at $1.5 \mathrm{~V}$ of bias. This corresponds to a resistance value of $5.54 \mathrm{G} \Omega$. The higher resistance measured in a pure oxygen environment at a pressure 
level of 250 Torr relative to the value measured at normal atmospheric conditions is an indication of sensitivity of the thin film to oxygen content in the surrounding environment.

The variation of $\mathrm{TiO}_{2}$ film resistivity to control chamber pressure was studied. As shown in Figure $7, \mathrm{TiO}_{2}$ thin film resistors act as perfect pressure sensors at room temperature and $\mathrm{N}_{2}$ pressure varying from 1 Torr to 760 Torr. The resistance increased as the nitrogen pressure increased. The resistance values were $0.313 \mathrm{G} \Omega, 0.786 \mathrm{G} \Omega, 1.078 \mathrm{G} \Omega$, and $1.72 \mathrm{G} \Omega$, at a pressure of 1 Torr, 10 Torr, 100 Torr, and 760 Torr, respectively.

In addition, $\mathrm{TiO}_{2}$ thin film resistors are sensitive to $\mathrm{O}_{2}$ pressure varying from 0.1 Torr to 100 Torr, with signal saturating above 100 Torr indicating sensor selectivity to $\mathrm{O}_{2}$ at room temperature. The resistance increased as the oxygen pressure increased. The resistance values were $0.533 \mathrm{G} \Omega, 2.132 \mathrm{G} \Omega$, and 4.000G $\Omega$, and 6.993G $\Omega$, at a pressure of 0.1 Torr, 1 Torr, 10 Torr, and 100 Torr, respectively. After 100 Torr, the measured I-V overlapped indicating saturation in the output signal. It is also observed that the resistance values at the same pressure are higher in the presence of the oxygen gas vs. nitrogen gas.

The transduction phenomenon of Titanium dioxide thin films and their oxygen sensitivity is a surface phenomenon. A structure with parallel $\mathrm{TiO}_{2}$ line resistors is proposed in order to maximize open surface area as shown in Figure 9.

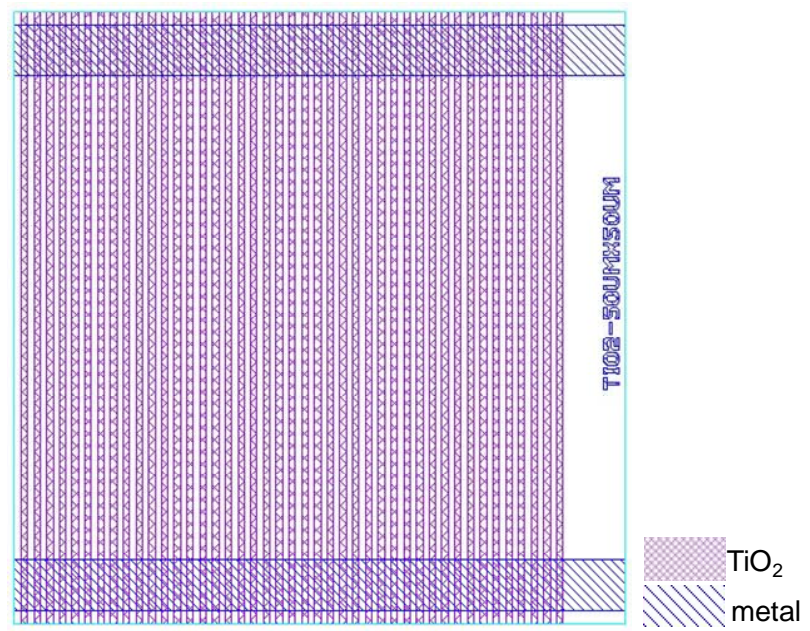

Figure 9: Layout schematic of a proposed $\mathrm{TiO}_{2}$ test structure with improved oxygen sensitivity.

\section{CONCLUSION}

A real-time hermiticity solution has been investigated for the first time. The proposed technique is based on the resistance measurement of a thin film Titanium dioxide resistor at a DC bias voltage as small as $1 \mathrm{~V}$. Titanium dioxide is an extrinsic semiconductor with some missing oxygen atoms in its crystalline structure. The variation of the oxygen concentration in the surrounding environment will modulate the resistivity of the thin film due to the change in the free carrier concentration. MEMS devices capped at sub-atmospheric pressure in an oxygen deficient environment will experience an abrupt change in built-in Titanium dioxide resistance as the film is exposed to atmospheric pressure and a $21 \%$ oxygen concentration. This is a clear indication of hermiticity and it can be used to monitor MEMS devices during operation versus the other techniques which check for hermiticity during manufacturing. Titanium dioxide thin films with metal electrode were built on insulated substrates, and their behavior to varying gas pressures and composition was studied in a control chamber. The testing results show clear distinction between resistance of Titanium dioxide films at room temperature at pressure versus environments with pure nitrogen gas and subatmospheric pressure levels. The thin films were very sensitive to both nitrogen and oxygen gas pressures in the range between 1 Torr to 760 Torr and 0.1 Torr to 100 Torr, respectively. In addition, the thin films were selective to oxygen with higher resistances measured at the same pressure level for oxygen gas as compared to nitrogen gas. A process flow to integrate the Titanium dioxide with the MEMS capping process is proposed using Through Silicon Via (TSV) technology with the thin film built on the cap side of the sensor. It is envisioned that this novel study will resolve many issues related to hermiticity by enabling in-use hermiticity monitoring in order to understand the root-cause of failures in field and part returns.

\section{ACKNOWLEDGEMENTS}

The authors would like to thank Dr. Noah Clay from the Harvard Center for Nanotechnology Systems (CNS) for his help in the fabrication and testing of the samples. The authors would also like to acknowledge Dr. Mehmet Hancer, Dr. Bill Sawyer, Dr. Li Chen, Dr. Christine Tsau, and Mr. Kieran Nunan from the Micromachined Product Division at Analog Devices for their valuable discussion and feedback.

\section{REFERENCES}

[1] F. Sammoura, A. Sparks, W. Sawyer, M. Bhagavat, M. Judy, and K. Yang, "Stiction in Low Humidity Environment," the Proceedings of $22^{\text {nd }}$ IEEE Micro Electro Mechanical Systems Conference, pp. 88-91, Sorrento, Italy, January 25-29, 2009.

[2] C. Goldsmith, T. H. Lin, W. R. Powers, and B. Norvell, "Micromechanical membrane switches for microwave application," IEEE MTT-S International Microwave Symposium Digest, pp. 91-94, Orlando, Fl, May 1996.

[3] R. R. Tummala, Microelectronics Packaging Handbook, Van Reinhold, New York, USA, 1989.

[4] G. Elger, L. Shiv, N. Nikac, F. Mueller, R. Liebe, M. Grigat, and Matthias Heschel, "Optical Leak Detection for Wafer Level Hermiticity Testing," IEEE/SEMI Int'l Electronics Manufacturing Technology Symposium, no. 29, pp. 326-331, San Jose, 2004.

[5] S. Pinter, "Method for Checking the Sealing of a Package and Apparatus for Measuring Viscosity," US Patent No. $5,872,309$.

[6] D. Lellouchi, J. Dhennin, X. Lafontan, D. Veyrie, J. F. Le Neal, and F. Pressecq, "A New Method for the Hermiticity Testing of Wafer-Level Packaging," Journal of Microelectronics and Microengineering, vol. 20, no. 2, February 2010.

[7] E. M. Logothetis and W. J. Kaiser, “TiO2 Film Oxygen Sensors Made by Chemical Vapor Deposition from Organometallics,” Sensors and Actuators, vol. 4, pp. 333-340, 1983.

[8] M. D. Stamme, "On the Dielectric Properties of DC Magnetron $\mathrm{TiO}_{2}$ Thin Films," Applied Surface Science, pp. 317-332, vol. 218, April 2003.

[9] A. A. Akl, H, Kamal, and K. Abdel-Hady, "Fabrication and Characterization of Sputtered Titanium Dioxide Films," Applied Surface Science, pp. 8651-8656, vol. 252, no. 24, October 2006.

\section{CONTACT}

*Firas Sammoura, 804 Woburn Street, Wilmington, MA 01887; Tel +1-781-937-2244; firas.sammoura@analog.com 